\title{
Hemophagocytosis in the Acute Phase of Fatal Kawasaki Disease in a 4 Month-Old Girl
}

\author{
Vehbi Doğan ${ }^{1}$, Erhan Karaaslan², Samet Özer², Rüveyda Gümüşer², Resul Y1lmaz² \\ 'Department of Pediatric Cardiology, Dr. Sami Ulus Maternity and Children Training and Research Hospital, Ankara, Turkey \\ ${ }^{2}$ Department of Pediatrics, Gaziosmanpaşa University School of Medicine, Tokat, Turkey
}

Background: Kawasaki disease is a systemic vasculitis predominately affecting coronary arteries. Hemophagocytic lymphohistiocytosis can complicate the course of Kawasaki disease. Rare cases of secondary hemophagocytic lymphohistiocytosis occurring during the acute phase of Kawasaki disease have been reported.

Case Report: We report here a 4 month-old girl with diffuse coronary ectasia and secondary hemophago- cytic lymphohistiocytosis occurring during the acute phase of incomplete Kawasaki disease.

Conclusion: Due to the large overlap in clinical symptoms, the presence of atypical findings for Kawasaki disease should suggest the possible diagnosis of hemophagocytic lymphohistiocytosis in these patients.

Keywords: Coronary artery dilatation, hemophagocytic lymphohistiocytosis, Kawasaki disease, macrophage activation syndrome
Kawasaki disease (KD) is a systemic vasculitis of unknown etiology predominately affecting medium-sized vessels such as the coronary arteries, which mainly involves infants and children (1). Hemophagocytic lymphohistiocytosis (HLH), is a systemic inflammatory disorder characterized by uncontrolled histiocytic proliferation, hemophagocytosis, macrophage activation, and up-regulation of inflammatory cytokines, which can complicate the course of KD (2-4). In most of the reported cases, HLH develop long after treatment or during the recurrent course of the disease (3-6). Herein we report a case with secondary HLH occurring during the acute phase of fatal $\mathrm{KD}$ in a 4 month-old girl.

\section{CASE PRESENTATION}

A previously healthy 4-month-old girl was admitted to a local hospital because of fever for 3 days and hospitalized for investigation and parenteral treatment. She was referred to our hospital because of prolonged fever and with a diagnosis of pneumonia at day 7 of febricity. Upon physical examination at admission, a fever of $39^{\circ} \mathrm{C}$, hepatosplenomegaly and a $2 / 6$ grading systolic ejec- tion murmur with normal chest auscultation findings were present. Her hemoglobin concentration was $9.6 \mathrm{gr} / \mathrm{dL}$ with a mean corpuscular volume of $77 \mathrm{fL}$, and her white blood cell (WBC) and platelet count were $25 \times 10^{3}$ and $919 \times 10^{3}$, respectively. Liver and kidney function tests were normal, C-reactive protein (CRP) level was $127 \mathrm{mg} / \mathrm{L}$ (range $0-5 \mathrm{mg} / \mathrm{L}$ ) and eryhtrocyte sedimentation rate (ESR) was $70 \mathrm{~mm} / \mathrm{h}$ (range 9-20) (Table 1). Chest $\mathrm{x}$-ray demonstrated no infiltration. She was consulted to pediatric cardiology because of murmur and echocardiography demonstrated a large secundum type atrial septal defect and moderate pulmonary valve stenosis with normal coronary artery structure. The detailed history and physical examination for classical findings of Kawasaki disease revealed mild oropharyngeal findings without any conjunctivitis, skin rash, peripheral extremity findings and typical lymphadenopathy. She had hepatosplenomegaly on admission and tests for the investigation of possible diagnosis of HLH were performed. Ferritin level was $386 \mathrm{ng} / \mathrm{mL}$, fibrinogen level was $544 \mathrm{mg} / \mathrm{dL}$ (range $238-498 \mathrm{mg} / \mathrm{dL}$ ), and total triglyceride and cholesterol levels were in the normal range with a value of 121 and $104 \mathrm{mg} / \mathrm{dL}$, respectively.

Fever continued and her clinical condition deteriorated in the following 3 days. The repeat blood tests demonstrated he- 

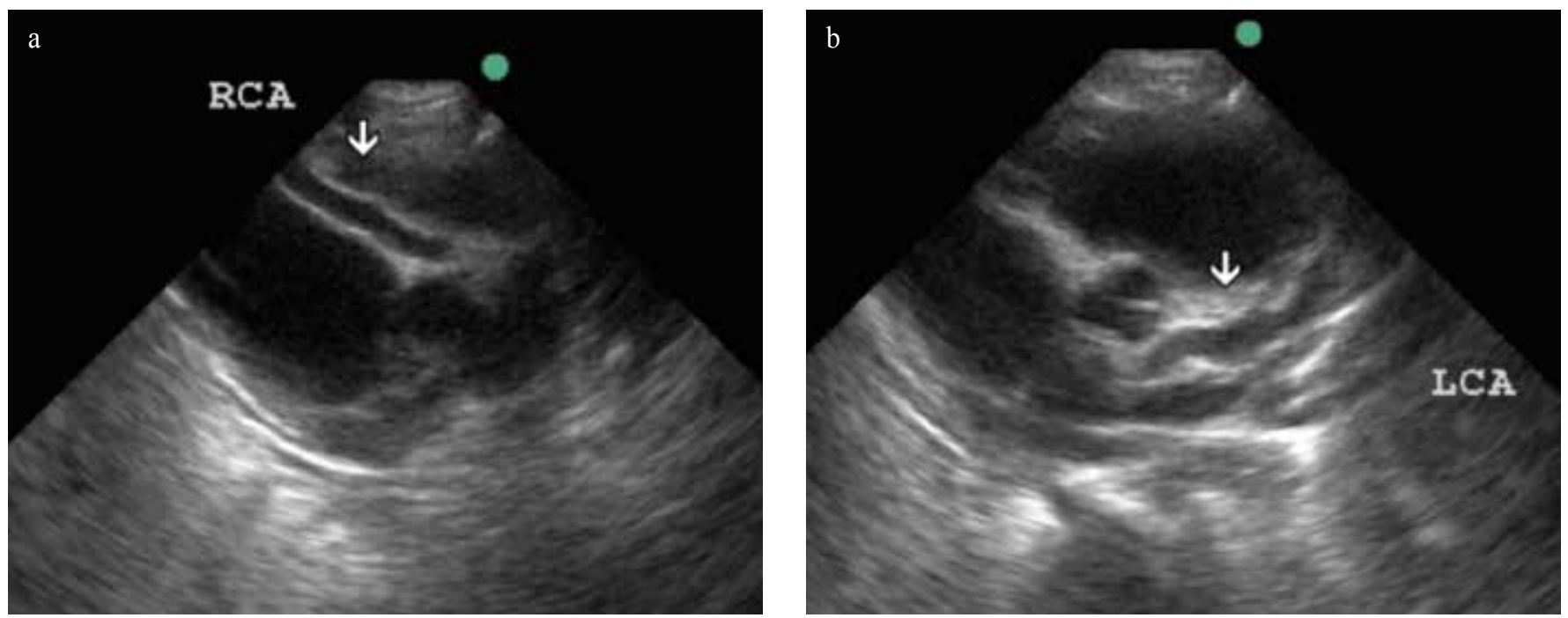

FIG. 1. a, b. Dilated right coronary artery with a diameter of $4.4 \mathrm{~mm}$ (a), dilated left coronary artery with an aneurysmal dilatation and a maximum diameter of $6.5 \mathrm{~mm}$ (b)

TABLE 1. Laboratory findings of the patient on admission (day 0 ) and $4^{\text {th }}$ day of hospitalization

\begin{tabular}{lccc}
\hline Test & Day 0 & Day 4 & Normal ranges \\
\hline Hemoglobin $(\mathrm{g} / \mathrm{dL})$ & 9.6 & 6.8 & $10.5-14$ \\
Hematocrit $(\%)$ & 29.3 & 20.1 & $32-42$ \\
White blood cell $\left(\mathrm{x} 10^{3}\right)$ & 25 & 26 & $6-14$ \\
Platelet $\left(\mathrm{x} 10^{3}\right)$ & 919 & 92 & $150-400$ \\
C-reactive protein $(\mathrm{mg} / \mathrm{L})$ & 127 & 158 & $0-5$ \\
Erythrocyte sedimentation rate $(\mathrm{mm} / \mathrm{h})$ & 70 & 13 & $9-20$ \\
Fibrinogen (mg/dL) & 544 & 199 & $238-498$ \\
Ferritin (ng/mL) & 386 & 1320 & $7-140$ \\
Alanine aminotransferase (U/L) & 8 & 28 & $13-45$ \\
Aspartate aminotransferase (U/L) & 35 & 129 & $10-40$ \\
Lactate dehydrogenase (U/L) & 484 & 436 & $180-430$ \\
Sodium (mmol/L) & 134 & 131 & $139-146$ \\
Albumin/Globulin (g/dL) & $3.2 / 2.5$ & $2.3 / 3.8$ & \\
Triglyceride (mg/dL) & 121 & 313 & $35-110$ \\
\hline
\end{tabular}

moglobin concentration of $6.8 \mathrm{gr} / \mathrm{dL}$, WBC count of $26 \times 10^{3}$, platelet count of $92 \times 10^{3}$, CRP of $158 \mathrm{mg} / \mathrm{L}$, and ESR of 13 $\mathrm{mm} / \mathrm{h}$ (range 9.4-20.2), aspartate aminotransferase of $129 \mathrm{U} / \mathrm{L}$ and $\mathrm{Na}$ of $131 \mathrm{mmol} / \mathrm{L}$, and albumin of $2.2 \mathrm{gr} / \mathrm{dL}$ (Table 1). Polymorphous skin rash developed during follow-up. A repeat echocardiogram was performed for KD and revealed diffuse coronary ectasia in both coronary arteries and fusiform dilatation with a maximum diameter of $6.7 \mathrm{~mm}$ and mild mitral regurgitation (Figure 1a, b). She was diagnosed as incomplete $\mathrm{KD}$ and intravenous immunoglobulin (Octagam: Octapharma AG; Lachen, Switzerland) with a dose of $2 \mathrm{~g} / \mathrm{kg}$ and heparin (Nevparine, Mustafa Nevzat İlaç Sanayi; İstanbul, Turkey) infusion was started on the $3^{\text {rd }}$ day of admission. Her fever subsided after intravenous immunoglobulin transfusion. Creatinine kinase $\mathrm{MB}$ fraction and troponin levels were within the normal limit, whereas ferritin levels increased to $1320 \mathrm{ng} / \mathrm{mL}$, fibrinogen levels decreased to $199 \mathrm{mg} / \mathrm{dL}$ and triglyceride levels increased to $313 \mathrm{mg} / \mathrm{dL}$. An additional diagnosis of HLH secondary to $\mathrm{KD}$ was considered in the light of these findings and hepatosplenomegaly. She died on the $5^{\text {th }}$ day of hospitalization shortly after realizing ST segment elevation attributed to possible myocardial infarction. The parents did not give permission for postmortem biopsy or bone marrow aspiration. Informed consent was obtained from the parents of the patient.

\section{DISCUSSION}

The diagnosis of KD is based on the clinical features of fever for at least 5 days together with at least 4 or 5 other features including rash, bilateral conjunctival injection, changes in peripheral extremities, lymphadenopathy and oropharyngeal changes (1). Our patient had only fever lacking the other features upon physical examination and a detailed history at admission to our hospital on the $7^{\text {th }}$ day of febricity. Later, during the follow-up, maculopapular skin rash was detected. The diagnosis of incomplete KD could be made after repeat echocardiogram demonstrating diffuse ectasia and saccular aneurysm of the coronary arteries. Our patient had fever, minimal oropharyngeal changes and skin rash in terms of classical KD findings. As we know that the incomplete form is found in 30$56 \%$ of the KD patients under 1 year of age, the presence of thrombocytosis at admission could be a warning sign for the diagnosis of $\operatorname{KD}(7,8)$. 
Our patient had hepatosplenomegaly and anemia at admission and later developed hyperferritinemia, hypertriglyceridemia, hypofibrinogenemia, hyponatremia and hypoalbuminemia, normal erythrocyte sedimentation rate with deepening anemia and decreasing platelet levels from $919 \times 10^{3}$ to $92 \times 10^{3}$ within 3 days of hospitalization. We were unable to measure the cytokine levels or natural killer cell activity in our center and perform postmortem investigations because of the absence of parental consent. However, according to the diagnostic criteria for HLH proposed in 2009, our patient meets the criteria for the diagnosis of HLH (2).

Hemophagocytic lymphohistiocytosis is an another cause of prolonged fever in children which has a large overlap of clinical symptoms with $\mathrm{KD}$; also, the co-existence of these two entities has been reported $(3-5,9,10)$. The mechanism by which HLH develops in these patients is unknown. Hypercytokinemia, as a result of abnormal immune-regulation, has been proposed to contribute to the pathogenesis of both diseases $(2,4)$.

In most of the published cases of HLH associated with KD, $\mathrm{KD}$ had a prolonged or recurrent course. Kang et al. found that the median interval between the first episode of $\mathrm{KD}$ and the onset of HLH was 13.3 days (4). Rare cases of secondary HLH occurring during the acute phase of KD have been reported $(9,10)$. Our patient had some features of HLH at admission, with a normal coronary artery structure detected by echocardiography. The second echocardiographic examination demonstrated coronary anomaly, suggesting that our patient had the coexistence of HLH and $\mathrm{KD}$, either HLH secondary to $\mathrm{KD}$, or both as a result of an as yet unknown common triggering mechanism.

In the literature, the age of patients with HLH secondary to $\mathrm{KD}$ ranges between 7 weeks to adolescence. Although KD is rare after the age of 5, a significant number of reported cases of HLH associated with KD cases are in children over 5 yearsold $(3,5,6)$. In most of these cases, HLH developed after a latent period after KD. Our patient was 4 months-old and HLH occurred in the acute phase of the disease. A similar presentation was reported by Titze et al. (9) and Chen et al. (10) in 7 week-old and 18 month-old patients, respectively. These findings may suggest older age as a predisposing factor for HLH and the younger ages for earlier onset HLH in KD patients.

Our patient died suddenly due to possible myocardial infarction on the $12^{\text {th }}$ day of febricity. Laboratory parameters indicative for severe HLH were lacking in our patient, so we did not attribute the cause of death to HLH. The two previously reported similar cases with HLH developing in the acute phase of KD also died because of myocardial infarction $(9,10)$. On the other hand, HLH associated with KD occurring long after treatment or during recurrent course $\mathrm{KD}$ had a better prognosis, suggesting that the co-existence of these two entities give rise to poor prognosis.

In conclusion, due to the large overlap in clinical symptoms, both KD and HLH should be considered in children with overlapping symptoms. The presence of atypical findings for KD such as hepatosplenomegaly, hyperferritinemia, hypertriglyceridemia or prolonged fever should prompt the suspicion of a possible diagnosis of HLH in these patients. Repeated echocardiograms for the suspected diagnosis of KD may be helpful in atypical presentations.

\section{Ethics Committee Approval: N/A.}

Informed Consent: Written informed consent was obtained from the parents of the patient who participated in this study.

Peer-review: Externally peer-reviewed.

Author contributions: Concept - V.D., E.K., R.G.; Design - V.D., S.Ö.; Supervision - V.D., R.Y., E.K.; Resource - V.D., R.G., S.Ö.; Materials - V.D.; Data Collection and/or Processing - V.D., R.G.; Analysis and/or Interpretation - V.D., E.K., R.Y.; Literature Search V.D., R.G.; Writing - V.D., E.K.; Critical Reviews - R.Y., S.Ö.

Conflict of Interest: No conflict of interest was declared by the authors.

Financial Disclosure: The authors declared that this study has received no financial interest.

\section{REFERENCES}

1. Tizard EJ. Complications of Kawasaki disease. Current Paediatrics 2005;15:62-8. [Crossref]

2. Filipovich AH. Hemophagocytic lymphohistiocytosis (HLH) and related disorders. Hematology Am Soc Hematol Educ Program 2009;127-31. [Crossref]

3. Palazzi DL, McClain KL, Kaplan SL. Hemophagocytic syndrome after Kawasaki disease. Pediatr Infect Dis J 2003;22:6636. [Crossref]

4. Latino GA, Manlhiot C, Yeung RS, Chanal N, McCrindle BW. Macrophage activation syndrome in the acute phase of Kawasaki disease. J Pediatr Hematol Oncol 2010;32:527-31. [Crossref]

5. al-Eid W, al-Jefri A, Bahabri S, al-Mayouf S. Hemophagocytosis complicating Kawasaki disease. Pediatr Hematol Oncol 2000;17:323-9. [Crossref]

6. Gültekingil A, Bayhan T, Kara A, Şahin M, Bilginer Y, Özen S. Kawasaki disease in two cousins with atypical presentation: case report. Turkiye Klinikleri J Pediatr 2010;19:347-9.

7. Joffe A, Kabani A, Jadavji T. Atypical and complicated Kawasaki disease in infants. Do we need criteria? West $J$ Med 1995;162:322-7.

8. Tseng CF, Fu YC, Fu LS, Betau H, Chi CS. Clinical spectrum of Kawasaki disease in infants. Zhonghua Yi Xue Za Zhi 2001;64:168-73.

9. Titze U, Janka G, Schneider EM, Prall F, Haffner D, Classen CF. Hemophagocytic lymphohistiocytosis and Kawasaki disease: Combined manifestation and differential diagnosis. Pediatr Blood Cancer 2009;53:493-5. [Crossref]

10. Chen Y, Shang S, Zhang C, Liu T, Yang Z, Tang Y. Hemophagocytic lymphohistiocytosis at initiation of kawasaki disease and their differential diagnosis. Pediatr Hematol Oncol 2010;27:244-9. [Crossref] 